\title{
Narrow BP Active-RC Filters with Reduced Power and Sensitivity
}

\author{
${\text { Dražen Jurišić* } \quad \text { Neven Mijat }{ }^{*} \quad \text { George S. Moschytz }}^{* *}$
}

\begin{abstract}
This paper presents the low-sensitivity and low-power realization of narrow high-order band-pass (BP) active resistance-capacitance (RC) filters. It is known that filters using multiple-feedback structures have reduced sensitivities to passive component tolerances. We present a filter realization using cascaded "two-amplifier biquartic" $B P$ active filter blocks (CBQ), with reduced numbers of operational amplifiers. The "impedance tapering" and the gainsensitivity-product minimization decrease both passive and active sensitivities of biquartic blocks. As an example, the design procedure and the Schoeffler's sensitivity analysis, were performed for an $8^{\text {th }}$-order Chebyshev narrow-BP filter.
\end{abstract}

\section{INTRODUCTION}

The realization of narrow band-pass filters yields very high pole Q-factor values, resulting from the application of the low-pass-to-band-pass (LP-BP) transformation to an all-pole low-pass function. By increasing the filter order, the pole Q-factors increase even more. High pole Q-factors produce high filter-magnitude sensitivity to the variation of passive components. In this paper, we compare a cascade (CAS) structure [Fig. 1(a)], to the filters using a cascaded biquart (CBQ) structure [1] [Fig. 1(b)] in order to reduce high passive component sensitivity. The idea of using cascaded biquarts to reduce pole Qs and, hence, sensitivities, was first proposed in [2] and sensitivity was examined in details in [3].

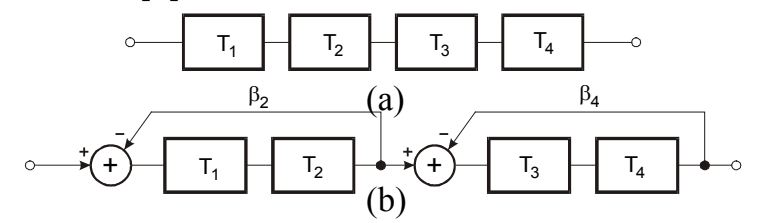

Figure 1: (a) CAS structure. (b) CBQ structure.

The power consumption is reduced by minimization of the overall number of operational amplifiers (opamps). We replace the commonly used multiamplifier realization of biquads by 1 -opamp biquads. Additionally, biquartic sections with two amplifiers

\footnotetext{
*Electronic Systems and Information Processing Dept., University of Zagreb, Unska 3, HR-10 000 Zagreb, Croatia, e-mail: [drazen.jurisic, neven.mijat]@fer.hr, tel: +3851612 99 49, fax: +38516129652.

** Bar-Ilan University, IL-52 900 Ramat-Gan, Israel, previously Swiss Federal Institute of Technology (ETH) Zürich, Switzerland, e-mail: moschytz@isi.ee.ethz.ch, tel: +97235317722, fax: +97235353567.
}

instead of three-amplifier sections are proposed. The filter magnitude sensitivity to the passive components (which we refer to as passive sensitivity) is further reduced by the use of low-sensitivity biquads of the CBQ, which are designed using the "impedance tapering" design method [4]. Additionally, the gainsensitivity-product (GSP [5]) of those biquads is minimized, reducing a filter's magnitude sensitivity to the open-loop gain $(A)$ variation of the active component. We refer to this as active sensitivity.

\section{THE HIGH-ORDER NARROW BP FILTER REALIZATION}

The $2^{\text {nd }}$-order building blocks (biquads) of the structures in Fig. 1, are commonly realized using "general purpose building blocks" (e.g. GP-2) [5]. The GP-2 section has low sensitivity to passive component variations (always less than unity) and it belongs to the "high-Q" circuits according to the classification in [5]. Its main disadvantage is that it requires a large number of opamps. For example, the $8^{\text {th }}$-order BP filter realized by the CBQ structure requires 3 opamps per each GP-2 biquad and 2 more for the summing amplifiers, i.e. a total of 14 opamps!

\subsection{Low-power band-pass 1-opamp biquads}

In order to reduce the power consumption, we use single-opamp biquads to build the $2^{\text {nd }}$-order BP blocks, and leave out the summing amplifiers in the CBQ. This reduces the opamp number in an $8^{\text {th }}$-order BP filter from 14 to 4 . We use Single-amplifier biquad (SAB) and Sallen-and-Key (SAK) biquads (with general impedance scaling factors $r$ and $\rho$ ) as shown in Figs. 2, and 3, respectively.

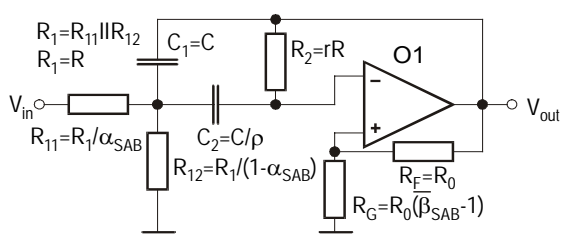

Figure 2: Deliyannis (SAB) 1-opamp-section.

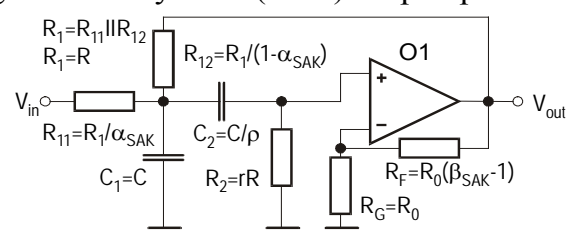

Figure 3: Sallen \& Key (SAK) 1-opamp-section.

The transfer function of the SAB (class-3; negative feedback) band-pass section in Fig. 2 is given by: 
$T(s)=\frac{V_{\text {out }}}{V_{\text {in }}}=\frac{-\alpha_{S A B} \bar{\beta}_{S A B} \cdot n(s)}{d(s)-\bar{\beta}_{S A B} \alpha_{S A B} n(s)}=\frac{K \cdot\left(\omega_{p} / q_{p}\right) \cdot s}{s^{2}+\left(\omega_{p} / q_{p}\right) \cdot s+\omega_{p}^{2}},(1)$

and the transfer function of the SAK (class-4; positive feedback) in Fig. 3 is given by:

$$
T(s)=\frac{V_{\text {out }}}{V_{\text {in }}}=\frac{\alpha_{S A K} \beta_{S A K} \cdot n(s)}{d(s)-\beta_{S A K}\left(1-\alpha_{S A K}\right) n(s)} .
$$

The filters in Figs. 2 and 3 have the same passive $R C$ network with the numerator and denominator given by:

$$
n(s)=k_{0} \omega_{0} s / q_{0} ; d(s)=s^{2}+\omega_{0} s / q_{0}+\omega_{0}^{2},(3)
$$

where $k_{0}=\frac{R_{2} C_{2}}{R_{1}\left(C_{1}+C_{2}\right)+R_{2} C_{2}} ; q_{0}=\frac{\sqrt{R_{1} C_{1} R_{2} C_{2}}}{R_{1}\left(C_{1}+C_{2}\right)+R_{2} C_{2}}$;

$$
\omega_{0}=\left(R_{1} C_{1} R_{2} C_{2}\right)^{-1 / 2} ; \text { and } R_{1}=R_{11} \| R_{12} \text {. }
$$

The gains $\bar{\beta}_{S A B}=1+R_{G} / R_{F}$ and $\beta_{S A K}=1+R_{F} / R_{G}$ represent the positive-feedback gain factors in class-3 and class-4 circuits, respectively. Note that the gains are related by $1 / \bar{\beta}_{S A B}+1 / \beta_{S A K}=1$. Both circuits are related by the complementary transformation [6], and the optimization conditions for the two, in terms of sensitivity, are identical. The attenuations $\alpha_{S A B}$ and $\alpha_{S A K}$ in both circuits are given by $\alpha=R_{12} /\left(R_{11}+R_{12}\right)$. Comparing (1) and (2) we obtain:

$$
\alpha_{S A B}=\alpha_{S A K} /\left(1-\alpha_{S A K}\right) ; \bar{\beta}_{S A B}=\beta_{S A K}\left(1-\alpha_{S A K}\right) \text {. }
$$

Consequently, if all passive elements of the SAK filter are known, we can use the shortcut way to calculate the passive elements of the SAB filter, and vice versa. We just need to calculate the values of the new $\alpha_{S A B}$ and $\bar{\beta}_{S A B}$ from the known $\alpha_{S A K}$ and $\beta_{S A K}$ using (5), or vice versa. In the new filter, passive elements such as $R_{1}, R_{2}, C_{1}$ and $C_{2}$ have the same values, whereas $R_{11}, R_{12}, R_{G}$ and $R_{F}$ readily follow from the new $\alpha$ and $\beta$.

\subsection{Sensitivity comparison of 1-and 3-amp biquads}

The disadvantage of the filters in Figs. 2 and 3 in comparison to the GP-2 section is that their magnitude sensitivity to passive components is proportional to the pole-Q factors. The sensitivity of the overall filter transfer function $F(s)$ to the component $x_{i j}$ variation is given by:

$$
S_{x_{i j}}^{F(s)}=S_{T_{i}(s)}^{F(s)} \cdot S_{a_{k}}^{T_{i}(s)} \cdot S_{x_{i j}}^{a_{k}},
$$

where $T_{i}(\mathrm{~s})$ represents the transfer function of the $i^{\text {th }}$ filter's $2^{\text {nd }}$-order sub-block and $a_{k}(k=0,1)$ represents its coefficients [where $a_{0}=\omega_{p}{ }^{2}, a_{1}=\omega_{p} / q_{p} ; \omega_{p}$ and $q_{p}$ are pole parameters defined in (1)]. The first factor in (6) is called structure-to-block sensitivity and it is dependent on the filter structure. Using multiple feedback structures this factor can be reduced, whereas in the cascade structure it equals unity. The second factor is called coefficient sensitivity and it depends exclusively on the generic filter type (e.g. Butterworth, Chebyshev, etc.) or, in other words, on the filter pole-Q values. Finally, the third factor represents the coefficient-to-component sensitivity and it depends on the way in which the filters were designed (e.g. "impedance tapering" design of the "medium-Q" filters). For the GP-2 sections the third sensitivity factor in (6) is always less than unity.

\section{2-OPAMP BIQUARTIC SECTIONS IN CBQ REALIZATION OF LOW-POWER BP FILTERS}

In [7][8] the two-amplifier version of biquart was first presented, in that feedback resistors replaced the summing opamps. The realization of two-amplifier biquarts using $\mathrm{SAB}$ and SAK sections are shown in Figs. 4 and 5, respectively.

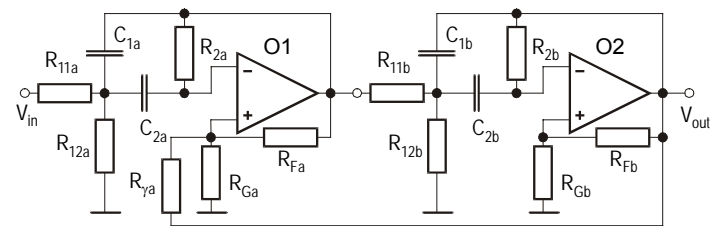

Figure 4: Biquart with SABs and feedback resistor.

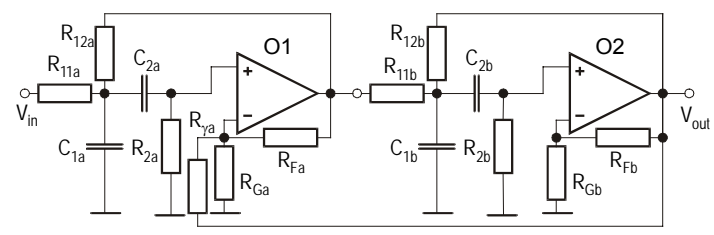

Figure 5: Biquart with SAKs and feedback resistor.

Each high-order (i.e. $n^{\text {th }}$-order; $n>2$ ) symmetrical BP transfer function can be written as a product of $4^{\text {th }}$-order factors if $n=4 k ; k=1,2, \ldots$ and eventually one $2^{\text {nd }}$-order factor if $n=2(2 k+1) ; k=1,2, \ldots$. The $4^{\text {th }}$-order factors have the form:

$$
F_{4}(s)=T_{1}(s) \cdot T_{2}(s)=\prod_{i=1}^{2} \frac{k_{i} \omega_{0 i} s / q_{i}}{s^{2}+\omega_{0 i} s / q_{i}+\omega_{0 i}^{2}}
$$

where $\omega_{01} \omega_{02}=\omega_{0}, q_{1}=q_{2}=q$, and $\omega_{0}$ is the center frequency of a BP filter. Equation (7) represents the cascade realization of a $4^{\text {th }}$-order BP filter.

It is well known that the overall sensitivity of a BP filter is minimal if each $4^{\text {th }}$-order factor defined by (7) is realized by a biquartic structure (in CBQ) with identical $2^{\text {nd }}$-order blocks [1]. The transfer function of a biquartic BP structure is:

$$
F_{4}(s)=\frac{T_{1 B} T_{2 B}}{1+b T_{1 B} T_{2 B}} ; T_{i B}(s)=\frac{k_{i B} \omega_{0} s / q_{B}}{s^{2}+\omega_{0} s / q_{B}+\omega_{0}^{2}},
$$

where $T_{i B}(s)(i=1,2)$ represent the transfer function of each $2^{\text {nd }}$-order BP sub-network, and $b$ represents a negative feedback factor. The design parameters for realizing the $4^{\text {th }}$-order biquartic structure from a given $4^{\text {th }}$-order cascade structure follows from equating (7) and (8). The design parameters are:

$$
\begin{gathered}
q_{B}=2 q \omega_{0} /\left(\omega_{01}+\omega_{02}\right) ; k_{1 B} k_{2 B}=k_{1} k_{2} q_{B}^{2} / q^{2} ; \\
b k_{1 B} k_{2 B}=\left(4 q^{2}-1\right) \cdot\left(\omega_{01}-\omega_{02}\right)^{2} \cdot\left(\omega_{01}+\omega_{02}\right)^{-2} .
\end{gathered}
$$

In [7][8] it is shown that if we leave out a summing amplifier and introduce a feedback resistor $R_{\gamma}$ to 
realize the two-amplifier biquart, the resulting network has two identical $2^{\text {nd }}$-order blocks with higher Q-factors, $q_{N}$, that readily follow from:

$$
\left(q_{N} / q_{B}\right)^{2}-2 \frac{q_{B}}{2 q_{B}-X q_{0}}\left(q_{N} / q_{B}\right)+\frac{q_{0}}{2 q_{B}-X q_{0}}=0 \text {, }
$$

where $X=1+b k_{1 B} k_{2 B}$ is defined by (9c), and $q_{0}$ is the passive pole $\mathrm{Q}$ defined by (4). Finally, with a known value of $q_{N}$, the feedback coefficient $\gamma_{1 \mathrm{~N}}$ follows from

$$
\gamma_{1 N}=2\left(q_{N} / q_{B}-1\right) /\left(\beta_{1} k_{2 B}\right),
$$

where $\beta_{1}$ represents the gain $\beta$ of the $1^{\text {st }}$ biquad section in the biquart, and $k_{2 \mathrm{~B}}$ is the pass-band gain of the $2^{\text {nd }}$ biquad section. Eqs. (10) and (11) hold for both SAB and SAK biquarts. Note that, because $q_{N}>q_{B}$ the resistance ratio $\gamma_{1 N}$ in (11) is always positive.

In the design process of 2-amplifier biquarts in Figs. 4 and 5, we use the impedance scaling factors (defined in the SAK and SAB above) and given by:

$$
R_{2}=r R_{1} ; \quad C_{2}=C_{1} / \rho \text {. }
$$

For both $\mathrm{SAK}$ and $\mathrm{SAB}$ biquads with known $k_{1 \mathrm{~B}}$, $\omega_{0}$ and $q_{N}$, the step-by step design procedure follows: (i) Choose capacitor $C$, and impedance scaling factors $\rho=1$, and $r>1$ and then calculate $R$ :

$$
R=\left(\omega_{0} C\right)^{-1} \sqrt{\rho / r} .
$$

(ii) Calculate the values

$$
q_{0}=\sqrt{\rho r} /(1+\rho+r), k_{0}=r /(1+\rho+r),
$$

and from (1)-(5) above calculate the $\alpha$ s and $\beta$ s from:

$$
\begin{gathered}
\bar{\beta}_{S A B}=1+(1+\rho) / r-q_{N}^{-1} \sqrt{\rho / r} ; \\
\beta_{S A K}=\beta_{S A K} \alpha_{S A K}+\bar{\beta}_{S A B}=k_{1 B} q_{0} /\left(k_{0} q_{N}\right)+\bar{\beta}_{S A B} ; \\
\alpha=k_{1 B} q_{0} /\left(k_{0} q_{N} \beta\right) .
\end{gathered}
$$

The above design equations are readily obtained by introducing (12) into (4). Note that when we write $\alpha$ and $\beta$ without subscripts they represent the gain and attenuation of both the SAB and SAK filter sections. (iii) The component values follow from:

$$
R_{1}=R ; R_{11}=R_{1} / \alpha ; R_{12}=R_{1} /(1-\alpha) ; C_{1}=C ; C_{1}=C / \rho
$$

(iv) Finally, choose $R_{G}$ and using feedback-factor $\gamma_{1 N}$, from (11), compute $R_{F}$ and $R_{\gamma}$ using:

$$
R_{F}=R_{G}\left[\beta_{S A K}\left(1-\gamma_{1 N}\right)-1\right] ; R_{\gamma}=R_{F} /\left(\beta_{S A K} \gamma_{1 N}\right) \text {, }
$$

where, for SAB filter design, we have:

$$
\beta_{S A K}=\bar{\beta}_{S A B} /\left(\bar{\beta}_{S A B}-1\right) \text {. }
$$

To design the circuit with min. GSP according to [5], (for both SAB and SAK) we modify the step (i) into: $(i$ ) choose $r>1$ and then calculate $\rho$ using:

$$
\rho=r /\left(36 q_{N}^{2}\right) \cdot\left[\sqrt{1+12 q_{N}^{2}(1+1 / r)}+1\right]^{2} .
$$

By this we reduce both passive (by choosing $r>1$ ) and active sensitivities. We can calculate the GSP:

$$
\Gamma_{S A K}=q_{p} \beta_{S A K}^{2}\left(1-\alpha_{S A K}\right) \sqrt{r / \rho} ; \Gamma_{S A B}=q_{p} \bar{\beta}_{S A B}^{2} \sqrt{r / \rho} \text {. }
$$

The $q_{0}$ in (10) has to be known in advance. If it were calculated according to the min. GSP criterion for SAB and SAK circuit, this would complicate the procedure for finding $q_{N}$, because the optimum $q_{0}$ depends on the value of $q_{N}$ as well. The problem is easily solved using a simple computer program. This problem does not arise (e.g. in the "impedance tapering" case) if we choose $r$ and $\rho$ in advance and they do not change their values until the end of the calculation.

\section{EXAMPLE}

As an example consider an $8^{\text {th }}$-order Chebyshev narrow-band BP filter with normalized bandwidth $B=0.1$, center frequency $\omega_{0}=1$, and reflection coefficient $\rho=10 \%$, (the pass-band ripple $R_{p}=0.044$ $\mathrm{dB})$. Its transfer function magnitude $\alpha(\omega)=20 \log$ $|F(j \omega)|[\mathrm{dB}]$ is shown in Fig. 6(a). The normalized transfer function parameters of the structures in Fig. 1 and their $2^{\text {nd }}$-order blocks $T_{i}(s)$ are given in Table 1 . Note that the gain values $k_{i}$ and feedback values $\beta_{i}$ $(i=1, \ldots, 4)$ are optimized by the method given in [9], to provide max. dynamic range. The frequency responses at all opamp outputs are shown in Fig. 6(b).

\begin{tabular}{|c|c|c|c|c|c|}
\hline & $i$ & $q_{p i}$ & $\omega_{p i}$ & $k_{i}$ & $\beta_{i}$ \\
\hline & 1 & 13.202 & 0.9755 & 1.0000 & \\
CAS & 2 & 13.202 & 1.0251 & 1.4274 & \\
& 3 & 31.919 & 0.9420 & 2.1733 & \\
& 4 & 31.919 & 1.0615 & 7.1188 & \\
\hline & 1 & 13.198 & 1.0000 & 1.3767 & \\
CBQ & 2 & 13.198 & 1.0000 & 1.0363 & 0.2991 \\
& 3 & 31.862 & 1.0000 & 4.4167 & \\
& 4 & 31.862 & 1.0000 & 3.4903 & 0.9402 \\
\hline
\end{tabular}

Table 1: Parameters of filter structures in Fig. 1.

\begin{tabular}{|c|c|c|c|c|c|}
\hline & $i$ & $q_{N}$ & $\omega_{p}$ & $k_{i B}$ & $\gamma_{i N}$ \\
\hline 1$)$ & 1 & 13.2714 & 1.0000 & 1.3899 & 0.00353542 \\
$r=1$ & 2 & 13.2714 & 1.0000 & 1.0379 & \\
$\rho=1$ & 3 & 34.5044 & 1.0000 & 4.8090 & 0.0141851 \\
& 4 & 34.5044 & 1.0000 & 3.7593 & \\
\hline
\end{tabular}

Table 2: Parameters of filter biquarts as in Fig. 5.

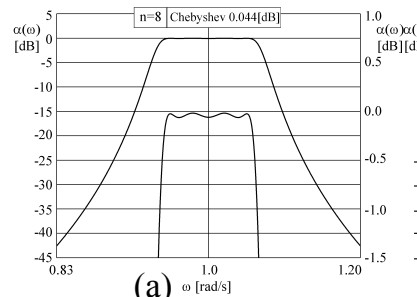

(a) $\stackrel{1.0}{1 \text { [rads] }}$

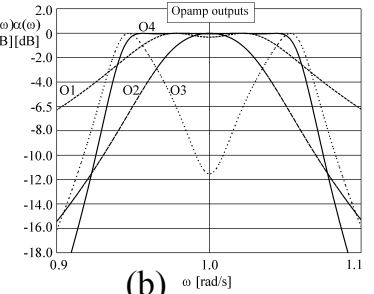

(b) ${ }_{0}^{1.0}[$ [rads]
Figure 6: (a) Frequency response of filter example.

(b) Frequency responses at section outputs.

In what follows we design the $8^{\text {th }}$-order BP filter using 2-opamp cascaded biquarts with SAK as in Fig. 5. To design the filters we apply various ways of impedance tapering to the SAK biquads, in the CAS and CBQ structures, i.e.: 1) $r=1, \rho=1$; 2) $r=4, \rho=4$; 3) $r=4, \rho=1$; 4) $r=1, \rho=4$; and 5) $r=4$, $\rho$ for min. GSP. After the transformation from 3- to 2-opamp biquarts a new optimization is required to provide maximum dynamic range, i.e. we have to calculate new gain values $k_{i B}(i=1, \ldots, 4)$, and new values of negative feedback factors $\gamma_{i N}(i=1,3)$ using (11). The new design parameters in the case 1) are in Table 2. 

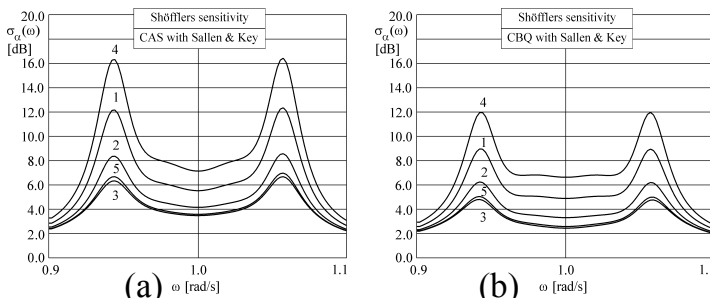

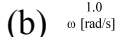

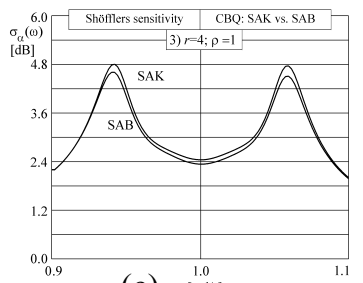

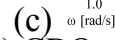

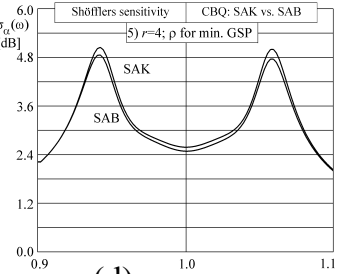

(d) $\underset{1.0}{1.0}$

Figure 7: Shöffler sensitivity of filters examples. (a) CAS. (b) CBQ. (c)-(d) Min. sensitivity SAK vs. SAB.

In a sensitivity analysis we assume the relative changes of the biquad resistors and capacitors to be uncorrelated normal random variables, with zeromean and 1\% standard deviation. The corresponding standard deviation $\sigma_{\alpha}(\omega) \quad[\mathrm{dB}]$ (related to the Shöfflers sensitivities) of the variation of the $\log$ gain $\Delta \alpha=8.68588 \Delta|F(j \omega)| /|F(j \omega)|$, with respect to passive elements, for CAS and CBQ structures for all design cases from 1) to 5) are in Figs. 7(a) and (b), respectively. The influence of feedback resistors on sensitivity was not considered.

Observing the standard deviation $\sigma_{\alpha}(\omega)[\mathrm{dB}]$ in the Fig. 7(a) one can investigate which is the proper impedance tapering design strategy of biquads in the cascade, in order to reduce the overall sensitivity of the $8^{\text {th }}$-order BP filter. Obviously the "ideally impedance-tapered" biquads (no. 2) and even more the "partially-tapered"1 biquads with equal capacitors (no. 3), decrease sensitivity with respect to component variations, compared to the non-tapered standard circuit (no. 1). The R-tapered biquads in the case no. 5 with $\rho$ for min. GSP shows somewhat higher passive sensitivities than those for the case no. 3. Further desensitization is obtained by an application of a negative feedback, which has a CBQ structure as in Fig. 1(b). The sensitivity curves of CBQ filters, designed with all five impedancetapering strategies applied to biquads, are shown in Fig. 7(b). The sensitivity curves in Fig. 7(b) have the same order as those in the CAS case in Fig. 7(a), i.e. $4,1,2,5,3$, starting from the curve with highest and ending with the lowest sensitivity. Note, that all CBQ curves in Fig. 7(b) are lower than those for the CAS structure in Fig. 7(a).

The Schöffler sensitivities of the CBQs with SAB and SAK section for the design strategies 3) and 5) are presented separately in Fig. 7(c) and (d), respectively. In both cases $\mathrm{SAB}$ biquads produce slightly lower sensitivity than SAK, although both circuits have the same passive-RC networks. Moreover, SAB has somewhat lower active sensitivity, which is apparent if (5) is substituted into (22). In a future full paper we'll include the effects of variations in the feedback resistors and in the amplifier open-loop gains, reducing the $\mathrm{Q}$ using

\footnotetext{
${ }^{1}$ The terms "ideal tapering" and "partial tapering" are used according to the definition in [4].
}

special approximating functions with low Qs, investigate tuning of biquarts, etc.

\section{CONCLUSIONS}

This paper presents a low-sensitivity and low-power realization of narrow high-order BP-filters. We present the detailed step-by-step design of lowpower filters with two-amplifier cascaded biquarts (CBQ). Furthermore, by application of a recently introduced "impedance tapering" design method of biquads, their passive sensitivities are additionally decreased. In this paper, we therefore present a twofold sensitivity reduction, one by introduction of negative feedback (application of the CBQ structure) and the other by the proper design of the $2^{\text {nd }}$-order sub-circuit building blocks in CBQ. The optimum design strategy that reduces both passive and active sensitivities is tapering the resistors and calculating the capacitor values for minimum GSP.

By application of the complementary transformation it is demonstrated that the realization of optimized biquarts using Sallen and Key also provides optimized Deliyannis biquads, whereas the latter shows slightly lower passive and active sensitivities.

\section{References}

[1] N. Mijat and G. S. Moschytz, 'Sensitivity of Narrowband Biquartic BP Active Filter Block', In Proc. $5^{\text {th }}$ ISYNT, Sarajevo, 1984, pp.158-163.

[2] J. Tow, "Some Results on Two-Section Generalized FLF Active Filters," IEEE Tran. on Circuits and Systems, Vol. CAS-25, pp. 181-184, April 1978.

[3] A. N. Gonuleren, "Sensitivity Analysis and Dynamic Range of Quad Filters and Some Comparative Results,” Int. J. Circuit Theory and Applications, Vol. 10, pp. 175-200, July 1982.

[4] G. S. Moschytz, "Low-sensitivity, low-power, active-RC allpole filters using impedance tapering," IEEE Trans. on CAS-II: vol. CAS-46, no.8, pp.1009-1026, Aug. 1999.

[5] G. S. Moschytz and P. Horn, Active Filter Design Handbook, Chichester, U.K.: Wiley 1981.

[6] G. S. Moschytz and P. Horn, "Optimizing two commonly used active-filter building blocks using the complementary transformation," Electronic Circuits and Systems, vol. 1, no. 4, pp. 125-132, July 1977.

[7] N. Mijat, V. Čosić and M. Vučić, "Design of Two-Amplifier Biquartic BP Active Filter Blocks", In Proc. $37^{\text {th }}$ Intl. Annual Gath. KoREMA, Zagreb, Hr, April 26-29, 1992, pp. 448-451.

[8] N. Mijat and V. Čosić, "Two-Amplifier Biquartic BP Active Filter Blocks with S\&K Sections", In Proc. $38^{\text {th }}$ Intl. Annual Conf. KoREMA, Zagreb, Hr, April 26-28, 1993, pp. 553-556.

[9] D. J. Perry, "Scaling Transformation of Multiple-Feedback Filters", Proc. IEE, vol.128, pp.176-179, Aug. 1981. 